\title{
ПРЕДСКАЗАНИЕ КОЭФФИЦИЕНТОВ РАСПРЕДЕЛЕНИЯ В СИСТЕМАХ С ОБРАЗОВАНИЕМ В ОРГАНИЧЕСКОЙ ФАЗЕ ВОДОРОДНОЙ СВЯЗИ МЕЖДУ РАСТВОРИТЕЛЕМ И РАСПРЕДЕЛЯЕМЫМ ВЕЩЕСТВОМ
}

Во многих работах по равновесию жидкость-жидкость установлена эмпирическая зависимость коэффициента распределения от физических свойств экстрагента и распределяемого вещества. Многократно указано также на роль водородной связи между экстрагентом и распределяемым веществом $\left[{ }^{1-6}\right]$, но пока оценка влияния ее на равновесие жидкостьжидкость носит скорее всего качественный характер. В настоящей работе на примере распределения фенола и крезолов между водой и простыми эфирами сделана попытка предсказать экстрагирующую способность растворителей на основании их склонности образовывать в неполярном растворителе Н-связи с распределяемым веществом. При этом предполагается, что растворы неассоциированного мономера и соединения фенолэфир в экстрагенте описываются теорией регулярных растворов.

\section{Распределение фенолов между фазами воды и протоноакцепторного растворителя}

Распределение вещества А между водой и протоноакцепторным растворителем В, взаимодействующим с ним посредством Н-связи, можно рассматривать как результат двух равновесных процессов:

1) Распределение не связанного в комплекс мономера

$$
\mathrm{A}_{(\mathrm{B})} \rightleftarrows \mathrm{A}_{(\mathrm{B})}
$$

с коэффициентом распределения

$$
\mathrm{K}_{\mathrm{A}}=\frac{\mathrm{x}_{\mathrm{A}(\mathrm{B})}}{\mathrm{X}_{\mathrm{A}(\mathrm{B})}},
$$

где $\mathrm{x}_{\mathrm{A}}$ - концентрация (в мол. долях) мономера А, индексы (в) и (в) относятся, соответственно, к фазам растворителя и воды.

2) Комплексообразование посредством Н-связи в органической фазе между А и В

$$
\mathrm{A}_{(\mathrm{B})}+\mathrm{nB}_{(\mathrm{B})} \rightleftharpoons \mathrm{AB}_{\mathrm{n}(\mathrm{B})}
$$

с константой равновесия

$$
K_{A B_{n}}=\frac{a_{A B_{n}(B)}}{a_{A(B)} \cdot a_{B(B)}^{n}}=\frac{x_{A B_{n}(B)} \cdot \gamma_{A B_{n}(B)}}{x_{A(B)} \cdot x_{B(B)}^{n} \cdot \gamma_{A(B)} \cdot \gamma_{B(B)}^{n}},
$$




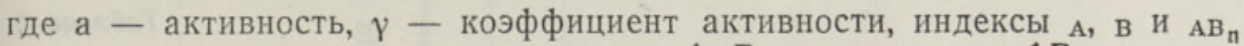
относятся, соответственно, к веществам A, В и соединению $\mathrm{AB}_{\mathrm{n}}$.

Суммарный коэффициент распределения $\mathrm{P}_{\mathrm{A}}$ выражается

$$
\mathrm{P}_{\mathrm{A}}=\frac{\mathrm{x}_{\mathrm{A}(\mathrm{B})}+\mathrm{x}_{\mathrm{AB}} \mathrm{B}_{\mathrm{n}}(\mathrm{B})}{\mathrm{x}_{\mathrm{A}(\mathrm{B})}}
$$

откуда с учетом (1) и (2) получим

$$
\mathrm{P}_{\mathrm{A}}=\mathrm{K}_{\mathrm{A}}\left(1+\mathrm{K}_{\mathrm{AB}_{\mathrm{n}}} \cdot \mathrm{x}_{\mathrm{B}(\mathrm{B})}^{\mathrm{n}} \frac{\gamma_{\mathrm{A}(\mathrm{B})} \cdot \gamma_{\mathrm{B}(\mathrm{B})}^{\mathrm{n}}}{\gamma_{\mathrm{AB}_{\mathrm{n}}(\mathrm{B})}}\right) \text {. }
$$

Обычно одноатомные фенолы образуют с простыми эфирами соединения состава $\mathrm{AB}$. Тогда при бесконечном разбавлении распределяемого вещества $\left(\mathrm{x}_{\mathrm{B}(\mathrm{B})} \rightarrow 1, \gamma_{\mathrm{B}(\mathrm{B})} \rightarrow 1\right)$

$$
\mathrm{P}_{\mathrm{A}}=\mathrm{K}_{\mathrm{A}}\left(1+\mathrm{K}_{\mathrm{AB}} \frac{\gamma_{\mathrm{A}(\mathrm{B})}}{\gamma_{\mathrm{AB}(\mathrm{B})}}\right) \text {. }
$$

По [7] распределение мономерной формы фенола между неполярными растворителями и водой хорошо поддается описанию теорией регулярных растворов при условии, что взаимодействие между распределяемым веществом и водой учитывается путем введения эмпирического значения для параметра растворимости водной фазы. Тогда отклонение от теории (в сторону увеличения $\mathrm{P}_{\mathrm{A}}$ ), проявляющееся в случае протоноакцепторных растворителей, можно рассматривать как результат образования водородной связи между А и В в органической фазе, и коэффициент распределения мономера $\left(\mathrm{K}_{\mathrm{A}}\right)$ определяется согласно теории регулярных растворов

$$
\lg K_{A}=\frac{\gamma_{A(B)}}{\gamma_{A(B)}}=\frac{V_{A}}{2,3 R T}\left[\left(\delta_{B}+\delta_{B}-2 \delta_{A}\right)\left(\delta_{B}-\delta_{B}\right)-R T\left(\frac{1}{V_{B}}-\frac{1}{V_{B}}\right)\right]+\lg \frac{V_{B}}{V_{B}},
$$

где $\mathrm{V}$ - мольный объем, $\delta$ - параметр растворимости.

Константа образования Н-связи $\mathrm{K}_{\mathrm{AB}}$, выраженная через активности, очевидно, связана с «концентрационной» константой равновесия $\mathrm{K}_{\mathrm{AB}(\mathrm{S})} \equiv$ $\equiv \mathrm{x}_{\mathrm{AB}(\mathrm{S})} / \mathrm{x}_{\mathrm{A}(\mathrm{S})} \cdot \mathrm{x}_{\mathrm{B}(\mathrm{S})}$, определенной спектроскопическим методом в условиях бесконечного разбавления взаимодействующих веществ в неполярном растворителе $\mathrm{S}$,

$$
\mathrm{K}_{\mathrm{AB}}=\mathrm{K}_{\mathrm{AB}(\mathrm{S})}^{\prime} \frac{\gamma_{\mathrm{AB}(\mathrm{S})}}{\gamma_{\mathrm{A}(\mathrm{S})} \cdot \gamma_{\mathrm{B}(\mathrm{S})}} .
$$

\section{Экспериментальная часть и обработка результатов}

Использованные в работе растворители перед применением несколько раз промывались водой и ректифицировались. Методика очистки фенолов и определения коэффициентов распределения при бесконечном разбавлении описана в [7]. Температуру в опытах поддерживали при $20 \pm 1{ }^{\circ} \mathrm{C}$. Как правило, значения $\mathrm{P}_{\mathrm{A}}$ в параллельных опытах не отличались больше, чем на $2-3 \%$.

При расчете $\mathrm{K}_{\mathrm{A}}$ по уравнению (5) использовали значения параметра растворимости, найденные в [7]: фенол $-12,60$, , -крезол $-12,24, n$-крезол - 12,23, вода - 19,00. Параметры растворимости для эфиров (табл. 1) рассчитывали по [ $\left.{ }^{8}\right]$ из теплот испарения при $20^{\circ}$, полученных по Джиакалоне и Ватсону [9] из критических параметров. $\mathrm{T}_{\text {кр }}$ и ркр рассчитывали методом Герцога $\left[{ }^{10}\right]$ по парахору и температуре кипения.

Для расчета $\mathrm{K}_{\mathrm{AB}}$ использовали данные работы ['1'], полученные спектроскопическим методом при бесконечном разбавлении взаимодейст- 
Tаблица 1

Критические свойства и параметры растворимости (при $20^{\circ} \mathrm{C}$ ) для симметричных эфиров

\begin{tabular}{|c|c|c|c|c|c|}
\hline Эфир & $\begin{array}{c}\mathrm{T}_{\mathrm{kpp}}, \\
{ }^{\circ} \mathrm{K}\end{array}$ & $\begin{array}{l}\mathrm{p}_{\mathrm{Kp}}, \\
\text { aTM. }\end{array}$ & $\underset{\kappa a \Omega / s o \Omega b}{\Delta \mathrm{H}_{\pi c \pi},}$ & $\begin{array}{c}\mathrm{V}_{\mathbf{B}}, \\
\mathrm{c} \mathrm{s}^{3} / M O \Omega t\end{array}$ & $\begin{array}{c}\delta_{\mathbf{B}}, \\
\left(\kappa a \Omega / c M^{3}\right)^{1 / 2}\end{array}$ \\
\hline Пропиловый & 528,4 & 28,42 & 8840 & 136,9 & 7,77 \\
\hline Изопропиловый & 496,3 & 28,42 & 8070 & 140,4 & 7,31 \\
\hline Бутиловый & 583,8 & 23,53 & 11090 & 169,2 & 7,88 \\
\hline Амиловый & 629,4 & 20,17 & 13230 & 202.1 & 7,91 \\
\hline Изоамиловый & 590,3 & 20,17 & 12120 & 203,5 & 7,53 \\
\hline Гексиловый & 667,4 & 17,77 & 15290 & 234,7 & 7,92 \\
\hline Гептиловый & 698,5 & 15,91 & 17430 & 263,0 & 8,00 \\
\hline Октиловый & 727,1 & 14,48 & 19530 & 295,6 & 8,01 \\
\hline
\end{tabular}

вующих веществ в циклогексане. $\mathrm{K}_{\mathrm{AB}(\mathrm{S})}^{\prime}$ для эфиров до амилового рассчитывали по уравнению (2) из [1'] с помощью средних значений $\alpha_{i}$ и $\delta_{j}$, приведенных там же. Для эфиров с более длинной цепью $\alpha_{i}$ находили по

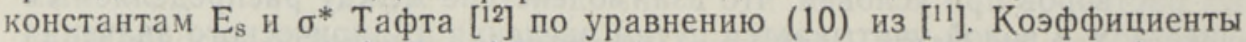
активности веществ A, В и АВ при бесконечном разбавлении в эфире и циклогексане рассчитывали, предполагая, что раствор подчиняется теории регулярных растворов. Для соединения АВ брали

$$
V_{A B} \cong V_{A}+V_{B} \text { и } \delta_{A B} \cong \frac{V_{A}}{V_{A B}} \delta_{A}+\frac{V_{B}}{V_{A B}} \delta_{B} .
$$

За исключением эфиров с разветвленной цепью, результаты расчета удовлетворительно сходятся с экспериментальными значениями $\mathrm{P}_{\mathrm{A}}$ (табл. 2). При этом, однако, для крезолов наблюдается небольшое систематическое отступление экспериментальных значений от рассчитанных в сторону увеличения. Нетрудно убедиться, что оно находится в пределах точности определения $\mathrm{K}_{\mathrm{AB}(\mathrm{S})}^{\prime}, \delta_{\mathrm{A}}, \delta_{\mathrm{B}}$ и $\delta_{\mathrm{B}}$.

Таким образом, предсказание равновесия жидкость-жидкость в системах с образованием водородной связи между растворителем В и распределяемым веществом А возможно при рассмотрении бесконечно разбавленного неидеального раствора А в В как регулярного трехкомпонентного $(\mathrm{A}+\mathrm{AB}$ в $\mathrm{B})$ и неидеального раствора $\mathrm{A}+\mathrm{B}$ в $\mathrm{S}$ как регуляр. ного четырехкомпонентного $(\mathrm{A}+\mathrm{B}+\mathrm{AB}$ в $\mathrm{S})$.

\section{Выводы}

1. Предложен метод для предсказания коэффициентов распределения протонодонорного вещества в системе вода-протоноакцепторный растворитель по параметрам растворимости и константе образования Н-связи в неполярном растворителе.

2. При проверке метода на примере распределения одноатомных фенолов между водой и алифатическими эфирами найдено удовлетвори гельное согласие теории и эксперимента. 


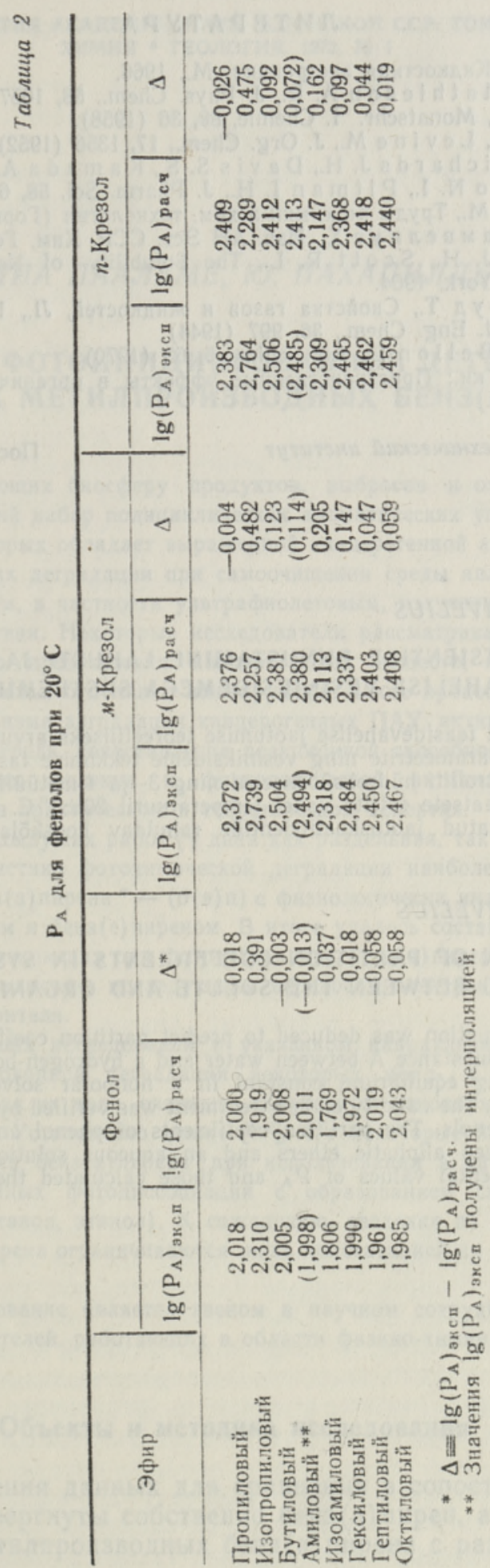




\section{ЛИТЕРАТ У РА}

1. Т р е й б а л Р., Жидкостная экстракция, М., 1966.

2. B r o w n C. P., Math i e s on A. R., J. Phys. Chem., 58, 1057 (1954).

3. S a n d e 11 K. B., Monatschr. f. Chemie, 89, 36 (1958).

4. P e a r s o n D. E., L e v in e M., J. Org. Chem., 17, 1356 (1952).

5. Higuchi T., Richards J. H., Davis S. S., Ka mada A., Hou J. P., Nakano M., N a k a no N. I., P itma n I. H., J. Pharm. Sci. 58, 661 (1969).

6. Корен м ан И. М., Труды по химии и хим. технологии (Горький), вып. 1, 54 (1970).

7. Мэлдер Л., Т а м в ел и у с Х., Изв. АН Эст. ССР, Хим. Геол., 21, 204 (1972).

8. Hildebrand J. H., Scott R. L., The Solubility of Nonelectrolytes, 3rd Edn, Dover, New York, 1964.

9. Рид Р., Ш е р в уд Т., Свойства газов и жидкостей, Л., 1971.

10. H e r z og R., Ind. Eng. Chem., 36, 997 (1944).

11. A b b oud J.-L., B ello n L., Ann. chim., 5, 63 (1970).

12. Т а ф т Р. У., В кн.: Пространственные эффекты в органической химии, М., 1960. c. 562 .

Таллинский политехнический институт

Поступила в редакцию

19/IX 1971

\section{MOLDER, H. TAMVELIUS}

\section{JAOTUSKOEFITSIENTIDE ENNUSTAMINE LAHUSTI JA JAOTUVA AINE VAHELISE VESINIKSIDEMEGA SUSTEEMIDES}

Esitatakse meetod faasidevahelise jaotumise teoreetiliseks arvutamiseks lahusti ja jao. tuva aine lahustuvusparameetrite ning vesiniksideme tekkimise tasakaalukonstandi alusel. Teooria kehtivust kontrolliti hüdroksübenseeni ning 3- ja 4-metüülfenoolide jaotumisel vee ja sümmeetriliste alifaatsete eetrite vahel temperatuuril $20 \pm 1^{\circ} \mathrm{C}$. Sedastati katseandmete ja teoreetiliselt arvutatud jaotuskoefitsientide rahuldav kooskōla.

\section{MOLDER, H. TAMVELIUS}

\section{PREDICTION OF PARTITION COEFFICIENTS IN SYSTEMS WITH H-BONDING BETWEEN THE SOLUTE AND ORGANIC SOLVENT}

A fundamental equation was deduced to predict partition coefficients $\left(\mathrm{P}_{\mathrm{A}}\right)$ of a hyd. rogen-bonding donor substance $\mathrm{A}$ between water and a hydrogen-bonding acceptor solvent $\mathrm{B}$ from the H-bonding equilibrium constant in a nonpolar solvent and the solubility parameters of $\mathrm{A}$ and $\mathrm{B}$. The validity of this treatment was verified by presenting an example of monofunctional phenols. The partition coefficients of phenol and $m$ - and $p$-cresols at infinite dilution between aliphatic ethers and an aqueous solution were determined at $20 \pm 1^{\circ} \mathrm{C}$. The experimental values of $\mathrm{P}_{\mathrm{A}}$ and those calculated theoretically are in good agreement. 